\title{
LÍNGUAS INDÍGENAS EM PAUTA NA AULA DE INGLÊS: POSSIBILIDADES PARA UMA EDUCAÇÃO LINGUÍSTICA CRÍTICA
}

\author{
INDIGENOUS LANGUAGES IN THE ENGLISH CLASS: \\ POSSIBILITIES FOR A CRITICAL LINGUISTIC EDUCATION
}

Isabela Ramalho Orlando*

\begin{abstract}
RESUMO
Neste trabalho é apresentada a experiência de um workshop sobre línguas indígenas brasileiras, realizado no contexto de um curso de inglês oferecido em uma universidade pública. Esses dados são parte de pesquisa sobre afetividade e ensino de inglês. Nesse sentido, buscou-se identificar e analisar os impactos afetivos que essa experiência produziu nos estudantes, a partir de suas produções textuais sobre o workshop. Compreende-se que as relações que se estabelecem entre sujeitos e objetos de conhecimento dependem, fundamentalmente, das práticas concretas de mediação desenvolvidas por agentes mediadores, as quais têm implicações tanto afetivas quanto cognitivas (LEITE, 2018). Assume-se uma visão discursiva da linguagem (BAKHTIN, 1929) e defende-se que o ensino-aprendizado de língua inglesa tem caráter formativo e deve considerar a natureza dialética da constituição do sujeito, da linguagem e da cultura, (ROCHA, 2012). Os dados indicam a aula de inglês como rica possibilidade para a discussão de temas sobre cultura, identidade e discurso. Com a experiência descrita neste trabalho - a discussão sobre línguas indígenas brasileiras, sobre a história dos povos indígenas e sua luta para preservação de suas línguas - os estudantes tiveram oportunidade de refletir sobre suas próprias língua e cultura e sobre seu processo de aprendizagem de inglês. A análise dos dados evidencia que as implicações desta experiência não cognitivas, mas também afetivas, podendo influenciar nas relações que se estabelecem entre estudantes e a língua inglesa, além de possibilitar uma aprendizagem crítica. Defende-se que o ensino de línguas deve tratar de temas como o descrito neste trabalho para que se alcance uma educação linguística antirracista, que, por sua vez, contribui para que se estabeleçam relações afetivas positivas com a língua estrangeira.
\end{abstract}

Palavras- chave: Afetividade; ensino de língua inglesa; ensino crítico de línguas; línguas indígenas.

\section{ABSTRACT}

This work presents the experience of a workshop on Brazilian indigenous languages, held in the context of an English course offered at a public university. These data are part of research on affect and English teaching. Therefore, one sought to identify and analyze the affective impacts that this experience produced on students, based on their textual productions about the workshop. It is understood that the relationships established between subjects and objects of knowledge depend, fundamentally, on the concrete mediation practices developed by mediating agents, which have both affective and cognitive implications (LEITE, 2018). A discursive view of language is assumed (BAKHTIN, 1929) and it is argued that the teaching and learning of the English language has a formative character and must consider the dialectical nature of the constitution of the subject, language and culture, (ROCHA, 2012). The data indicate the English class as a rich possibility for the discussion of topics on culture, identity and discourse. With the experience described in this work - the discussion about Brazilian indigenous languages, about the history of indigenous peoples and their struggle to preserve their languages - the students had the opportunity to reflect on their own language and culture and on their English learning process. The data analysis shows that the implications of this experience are not only of a cognitive, but also affective, which can influence the relationships that are established between students and the English language, in addition to enabling critical learning. It is argued that language teaching should deal with themes such as the one described in this work in order to achieve anti-racist linguistic education, which, in turn, contributes to establishing positive affective relationships with the foreign language.

Keywords: Affect; English language teaching; critical language teaching; indigenous languages.

\section{INTRODUÇÃO}

Dentre as muitas temáticas abordadas no ensino de línguas, dois temas vêm sendo destacados: as emoções e as questões étnico-raciais. Sobre o primeiro ponto, Swain (2013) indica que as emoções têm sido negligenciadas nas pesquisas sobre ensino-aprendizagem de línguas e, dados que elas têm implicações significativas neste processo, é preciso desenvolver estudos sobre a temática. Quanto ao segundo tópico citado, as questões étnico-raciais, muitos autores da linguística aplicada (OSLER e STARKEY, 2000; FERREIRA, 2007) têm destacado a necessidade de que as salas de aula de línguas se coloquem como espaços de enfrentamento ao racismo. Este trabalho colocará em pauta ambas questões, por meio da descrição e discussão da experiência de um workshop sobre línguas indígenas brasileiras realizado no contexto de um curso de inglês. Além de analisar as contribuições dessa atividade para a educação crítica dos estudantes, serão evidenciadas suas implicações afetivas para os alunos.

\footnotetext{
* Doutoranda em Educação pela Universidade Estadual de Campinas (Unicamp), Campinas, SP, Brasil. isabelarorlando@gmail.com Orcid: https://orcid.org/0000-0001-9721-2402
} 
Considera-se imprescindível a inserção de questões étnico-raciais nos processos educativos, uma vez que a cultura e a história dos povos indígenas e comunidades africanas têm sido sistematicamente apagadas dos currículos escolares (MOURA, 2005; FEITOSA, 2012). Segundo Moura (2005), a escola nega ao estudante o conhecimento sobre as diferentes etnias que contribuíram para formação da identidade brasileira. Tal situação é agravada pelo fato de história (parcialmente) contada nas instituições educativas apresentar apenas o ponto de vista dos vencedores, isto é, dos colonizadores (MOURA, 2005).

Munanga (2005) aponta os que professores são coniventes diante de casos de racismo e preconceito, ignorando casos de racismo nos quais sua intervenção seria fundamental para dar aos estudantes uma nova perspectiva sobre a diversidade e a valorização de outras culturas. Nesse sentido, as autoras Camargo e Ferreira (2012) defendem que, na sala de aula de língua inglesa, a língua seja vista como instrumento contra o discurso hegemônico.

Diante deste cenário, a discussão sobre as culturas e línguas indígenas se coloca como um dos temas que podem ser abordados, não esgotando as problemáticas possíveis de serem trabalhas na aula de língua inglesa. A experiência apresentada neste artigo é parte de uma pesquisa de mestrado, realizada em um centro de línguas universitário. Considerando que as relações entre sujeito e objeto de conhecimento não são somente cognitivas, mas também afetivas, a pesquisa buscou identificar como as práticas pedagógicas da professora de inglês contribuíram para a aproximação dos estudantes com a língua inglesa.

Este trabalho centra-se em uma das atividades de ensino promovidas pela professora - um workshop sobre programas de revitalização de línguas indígenas brasileiras. Inicialmente, será apresentado referencial acerca de ensino de línguas em uma perspectiva crítica, ressaltando-se o caráter formativo da aprendizagem de língua inglesa. Em seguida, discute-se a questão da afetividade, com base em Espinosa (1677), Vigotski (1934, 1960) e Wallon (1941, 1942). São abordadas também as especificidades da questão afetiva no processo de ensino-aprendizagem de língua. Por fim, por meio de produções textuais dos estudantes, é apresentada a experiência do workshop e são tecidas análise sobre as implicações afetivas desta vivência, bem como sobre suas contribuições para uma educação linguística crítica.

\section{ENSINO DE INGLÊS EM UMA PERSPECTIVA CRÍTICA}

A experiência de aprender uma língua estrangeira, especialmente a língua inglesa, tem sido associada a razões mercadológicas, como a esperança de conseguir um emprego, de passar no vestibular ou de se integrar a um mundo globalizado. Contrário a isso, Siqueira (2018) alerta que é preciso compreender que, ao ensinar línguas, o professor está, de fato, envolvido em um processo educativo e não apenas em uma prática utilitária na qual o comércio de ensino de línguas foi transformado.

Rocha (2012) e Rocha e Maciel (2013) defendem que o ensino-aprendizagem de línguas pode ter caráter formativo e contribuir para maior participação social. Para isto, é preciso que o ensino de línguas problematize as ideias de democracia e cidadania, levando em consideração os processos contemporâneos de globalização. Em diálogo com estes autores, compreende-se o ensino de língua inglesa em uma perspectiva crítica a partir de uma visão discursiva da linguagem, compreendendo-a como central na constituição dos sujeitos e da realidade (BAKHTIN, 1929).

Nessa perspectiva, não se pode conceber o inglês como língua neutra ou língua universal e é preciso entender os jogos de poder envolvidos no status que este idioma alcançou. Rodrigues (2011) destaca os processos ideológicos por meio dos quais se promove o inglês como língua global: naturalização da expansão da língua; a crença em sua universalidade; o apagamento das contradições de sua expansão, esquecendo que sua hegemonia é força de exclusão social e política de inúmeras outras línguas; produção de sentido teleológico da história, segundo o qual a língua inglesa estaria predestinada a tornar-se língua global. Na mesma direção, Pennycook (1994) defende que a expansão da língua inglesa no mundo significa o silenciamento de diversas culturas e povos.

Diante disso, defende-se que a aprendizagem da língua inglesa pode ter caráter educativo, desde que seja promovida em uma perspectiva crítica. Jorge (2009, p.163) afirma que esse caráter formativo reside "na possibilidade de que os estudantes se tornem mais conscientes da diversidade cultural que constitui o mundo". Autores como Rajagopalan (2004a) e Pennycook (1994) defendem que a sala de aula de língua inglesa seja um espaço em que se questionem discursos hegemônicos que colocam Inglaterra e Estados Unidos como referências deste idioma. 
Dessa forma, quando se fala em educação linguística crítica, o ensino da língua não se restringe ao ensino de fenômenos linguísticos, descolados de qualquer contexto significativo. Pelo contrário, em uma perspectiva crítica, a sala de aula de língua é espaço para discussões, para troca de experiências e para o questionamento de discursos hegemônicos. Siqueira (2018, p. 204) afirma que é na educação linguística crítica que

podemos ensinar línguas, visando, entre outras coisas, ao combate às desigualdades sociais e práticas de subalternização e opressão que tanto alimentam as tão comuns e naturalizadas relações assimétricas dos espaços sociais que habitamos.

Há um posicionamento claro de que a educação linguística crítica se coloca em favor da luta por justiça social. Rajagopalan (2003) aponta que o educador crítico deve instigar o questionamento da intocabilidade de certos dogmas. Entende-se que esses dogmas incluem, embora não se restrinjam a, as relações de gênero, raça e classe. Neste artigo, a discussão enfocará os fenômenos que envolvem raça/etnia, por estarem diretamente relacionadas à experiência que será descrita posteriormente (o que não significa reduzir a importâncias dos outros temas). No que se refere às questões de raça e etnia, Feitosa (2012) afirma que as relações raciais desiguais estão presentes no cotidiano escolar, uma situação que se agrava pelo fato de o currículo espaldar essa prática, já que favorece apenas o conhecimento que privilegia os padrões brancos de cultura.

Diante desse cenário, levanta-se a questão de qual seria o papel do ensino- aprendizagem de uma língua estrangeira no enfretamento do racismo. Osler \& Starkey (2000) defendem que qualquer curso, inclusive o de língua estrangeira, que procura construir um ensino para a democracia deve considerar o racismo como uma barreira para a participação integral na cidadania e necessita oportunizar que os estudantes explorem os assuntos de identidade. Ferreira (2007) argumenta que as disciplinas de línguas estrangeiras também carregam a responsabilidade de abordar tópicos que promovam igualdade em termos de raça/etnia. A autora destaca a necessidade de se repensar os propósitos da educação e de engajar os estudantes em atividades que favoreçam uma ação social transformadora da realidade e que haja promoção da igualdade racial e étnica. Para que tais ações sejam concretizadas, é preciso repensar a forma como os professores trabalharão com os alunos e a maneira como os professores são educados em cursos de formação.

Rajagopalan (2003) afirma que o ensino de inglês que pretende a emancipação do cidadão, além da aquisição de conhecimentos linguísticos, deve estar engajado com o ensino intercultural e deve considerar a origem, a cultura e o lugar social que o falante ocupa. Desta forma, é possível argumentar que experiências que oportunizem aos estudantes aprender e refletir sobre a história e cultura de seu próprio país é de extrema importância para a concretização de um ensino de língua numa perspectiva crítica. Argumenta-se que a experiência descrita neste artigo proporcionou, em alguma medida, tais reflexões e aprendizados.

\section{AFETIVIDADE E DESENVOLVIMENTO HUMANO EM UMA CONCEPÇÃO MONISTA}

No pensamento filosófico ocidental houve, durante séculos, o predomínio de visões dualistas sobre o ser humano, isto é, que supunham a cisão entre razão/emoção, afeto/cognição e corpo/mente. Leite (2012) adverte que assumir esta dicotomização significa afirmar que o ser humano ora pensa, ora sente, sem que haja relação entre estas duas dimensões. O autor observa ainda que, a partir do pensamento dualista, o fenômeno humano nunca poderia ser estudado por completo pela ciência, uma vez que os afetos, por serem tidos como parte anímica, não poderiam ser objeto de estudos científicos. Dessa maneira, somente uma parte do homem - a razão - poderia ser tema de investigações científicas. Destaca-se ainda o fato de que essas correntes filosóficas elegeram a razão como característica humana central, enquanto a emoção corresponderia ao lado nebuloso do ser humano (LEITE, 2012).

No entanto, o pensamento dualista tem sido confrontado ao longo do tempo e, nesse sentido, destacam-se os trabalhos de Espinosa ${ }^{1}$ (SPINOZA, 1677). Esse filósofo holandês do século XVII rompeu a hierarquia secular que compreende a alma como superior ao corpo ao defender que ambos são atributos de uma mesma substância e que seguem as mesmas leis, sem separação e hierarquia entre eles. Conforme Sawaia (2018, p.30), "nesta perspectiva, o afeto é uma condição corporal e, ao mesmo tempo, a ideia dessa condição, pois não há nada na mente que não tenha sido afecção do corpo". Sawaia $(2018$, p. 31) destaca ainda que, na teoria dos afetos de Espinosa, os pensamentos e

1. Encontram-se muitas grafias para o nome do filósofo, além de mudanças no seu primeiro nome (Bento ou Baruch, um de origem portuguesa e outro holandesa). Apesar de a bibliografia consultada utilizar a grafia Spinoza, optou-se por usar "Espinosa" ao falar sobre o autor, por ser esta a maneira utilizada por outros pesquisadores brasileiros (CHAUÍ, 1995; SAWAIA, 2018) 
ações humanos estão ligados "às experiências do corpo no decorrer da vida em sociedade", portanto há uma estreita relação entre afeto, ideia e ação. Para esse filósofo, os afetos diminuem ou aumentam a potência de ação dos seres humanos (SPINOZA, 1677). A alegria e a tristeza são os afetos primários e estão ligados à potência de ação dos sujeitos. A alegria aumenta a potência de agir e de pensar, enquanto a tristeza diminui esta potência (SPINOZA, 1677).

Séculos mais tarde, Vigotski $(1934,1960)$, pesquisador que atuou na União Soviética no início do século XX, se inspiraria em Espinosa para denunciar aquilo que considerava um dos maiores erros da Psicologia tradicional: a separação da dimensão intelectual da consciência de sua dimensão afetiva (VIGOTSKI, 1934). Assim como Espinosa, Vigotski $(1934,1960)$ defendia a indissociabilidade entre pensar, sentir e agir. Com base no método materialista histórico-dialético de Marx, Vigotski buscou explicar o desenvolvimento das funções psicológicas superiores, tipicamente humanas, como um processo histórico-cultural. O autor defende que a gênese do psiquismo é social e que a relação entre indivíduo e o mundo nunca é direta, mas mediada por sistemas simbólicos (VIGOTSKI, 1960)

A linguagem é considerada como sistema simbólico básico de todo grupo humano (LEITE, 2012). Nogueira (2010, p.61) lembra que "as relações interpsicológicas são mediadas pelos processos de significação e de produção de sentidos na/da linguagem, que constituem e são constituídos pelos homens". É importante destacar, especialmente no âmbito deste trabalho, que a linguagem ocupa espaço central na obra de Vigotski, tendo o autor se dedicado ao estudo da relação entre pensamento e linguagem. Segundo ele, estes dois fenômenos são complexos e, portanto, deveriam ser estudados a partir de unidades que não se decompõem e que se conservam. Vigotski (2009, p. 10) defende que esta unidade, na relação pensamento e linguagem, seria o significado da palavra, uma vez que este é igualmente "fenômeno da linguagem por sua natureza" e "fenômeno do campo do pensamento".

A discussão sobre pensamento e linguagem guarda também relação com a questão da afetividade. Magiolino e Smolka (2013), partindo da noção de significação (produção material, de natureza social, de signos e de sentidos), argumentam que os seres humanos não apenas sentem, mas "sentem com sentido e significado" (MAGIOLINO e SMOLKA, 2013, p. 106). Segundo as autoras, as emoções humanas são transformadas em uma produção histórica e cultural nas relações interpessoais, que são, por sua vez, mediadas por signos. Neste processo, evidencia-se o papel das palavras na viabilização do desenvolvimento das emoções e na possibilidade de que os seres humanos se tornem conscientes delas (MAGIOLINO e SMOLKA, 2013).

Ainda para a compreensão dos afetos na perspectiva da Teoria Histórico-Cultural, é válido trazer as considerações de Sawaia e Magiolino (2016), que, a partir da análise das obras de Espinosa e Vigotski, cunharam uma definição para o sentimento, o qual corresponderia a uma emoção que permanece presentificada. Este conceito "permite analisar as emoções desvinculadas das afetações imediatas que a provocaram e perpetuadas como uma experiência emocional intensiva" (SAWAIA e MAGIOLINO, 2016, p.84). O conceito de sentimento possibilita a compreensão de que os afetos estão sempre em movimento e que não precisam estar necessariamente ligados à afetação do instante, mas podem se manter como ambiência afetiva ou se sedimentarem em paixões tristes e alegres, que aumentam ou diminuem a potência de agir (SAWAIA e MAGIOLINO, 2016). Sawaia (2000, p.15) afirma, ainda, que afetividade (emoção e sentimento) é "o nome atribuído à capacidade humana de elevar seus instintos à altura da consciência, por meio dos significados, de mediar a afecção pelos signos sociais, aumentando ou diminuindo a potência de ação".

Outros autores também se dedicaram ao estudo da afetividade a partir de uma perspectiva monista, dos quais destacam-se os trabalhos de Wallon $(1941,1942)$, pesquisador francês que, assim como Vigotski $(1934,1960)$, adotou o método materialista histórico-dialético para conduzir suas investigações. Muitas foram as contribuições desse autor, contudo, neste artigo será enfocada a questão da afetividade e, mais especificamente, serão destacados os pontos de convergência com a Teoria Histórico-Cultural, descrita anteriormente.

Wallon $(1941,1942)$, de maneira semelhante a Vigotski $(1934,1960)$, dá ênfase ao papel das interações sociais para o desenvolvimento humano, além de apresentar uma visão integradora deste processo, segundo a qual o funcionamento humano integra quatro campos: motor, cognitivo e afetivo, que constituem o quarto campo, a pessoa. O primeiro oferece as funções responsáveis pelos movimentos do corpo e possibilita seu deslocamento pelo espaço, além disso, oferece estrutura para expressão das emoções e sentimentos. Já o conjunto cognitivo é composto por signos e é responsável "pela aquisição, transformação e manutenção do conhecimento" (MAHONEY e ALMEIDA, 2004, p. 18). 
O conjunto afetivo, por sua vez, sinaliza, por meio das emoções, dos sentimentos e da paixão, como o ser humano é afetado pelo mundo (MAHONEY e ALMEIDA, 2004). Wallon $(1941,1942)$ afirma que ser afetado pelo mundo estimula tanto os movimentos do corpo quanto a atividade mental, ou seja, há uma integração entre os campos funcionais. Em sua obra, a afetividade "refere-se à disposição do ser humano de ser afetado pelo mundo, por meio de sensações ligadas a tonalidades agradáveis ou desagradáveis" (ALMEIDA e MAHONEY, 2014, p. 17). Portanto, a afetividade engloba um conjunto amplo de manifestações que envolvem as emoções (origem biológica), os sentimentos (origem psicológica) e a paixão (ligada à ativação do autocontrole), e que se desenvolve através da apropriação dos sistemas simbólicos culturais.

Wallon $(1941,1942)$ considera que a afetividade exerce papel fundamental no desenvolvimento humano, sendo determinante tanto na construção da pessoa quanto na construção do conhecimento. Verifica-se que em todos os estágios de desenvolvimento estão presentes, de maneira indissociável, a afetividade e a cognição, observandose uma alternância de predomínio de cada uma delas. Com isto, destaca-se, novamente, que o autor concebe a afetividade a partir de uma visão monista, tal como Vigotski (1934).

Conforme já mencionado, Wallon $(1941,1942)$ dá grande relevância para as relações sociais ao estudar o desenvolvimento humano, afirmando que é na dialética entre Eu e Outro que se realiza a singularidade do indivíduo. Considera-se que trocas e construções estabelecidas na interação com o outro permitem ao indivíduo caracterizar-se como um sujeito único, com base na construção de um universo simbólico pessoal. Com isto, pode-se destacar outro ponto de convergência entre Wallon $(1941,1942)$ e Vigotski (1960): o entendimento de que a constituição do sujeito se dá na e pelas relações sociais.

Com base nestes dois autores, é possível compreender que todas as interações sociais são carregadas de conteúdos afetivos, sendo que a afetividade envolve vivências e formas de expressão humanas complexas e desenvolvese com a apropriação de sistemas simbólicos da cultura, principalmente a linguagem. Por envolver elementos simbólicos da cultura, a complexificação das manifestações afetivas só é possível por meio da mediação do outro.

\subsection{Afetividade na sala de aula de língua inglesa}

Tomando Espinosa, Vigotski e Walon como referenciais teóricos, os pesquisadores do Grupo do Afeto têm realizado, ao longo das últimas duas décadas, inúmeras pesquisas sobre afetividade nas práticas pedagógicas. Essas investigações possibilitaram um maior entendimento sobre as relações que se estabelecem entre sujeito e objeto de conhecimento, considerando-se que estas relações são de natureza tanto afetiva quanto cognitiva (LEITE, 2018). É válido relatar que este conjunto de pesquisas, embora abarquem temas diversos e tenham sido realizadas em diferentes contextos, seguiram alguns pressupostos em comum, tais como: a concepção de que o processo de apropriação de conhecimento pelo sujeito é sempre mediado, seja por pessoas físicas ou por agentes culturais; as relações entre sujeito e objeto de conhecimento são marcadamente afetivas, sendo que a qualidade da mediação vivenciada pelo sujeito é, em grande medida, determinante do tipo de relação que se estabelece entre aprendiz e objeto de conhecimento (LEITE, 2012).

Essas pesquisas evidenciaram que as decisões tomadas pelo professor no planejamento e desenvolvimento de seu curso têm implicações nessas relações entre estudantes e o objeto de conhecimento estudado. Conforme Leite (2012), algumas dessas decisões são: a escolha dos objetivos de ensino; o ponto de partida do ensino; a identificação e a organização dos conteúdos; a escolha dos procedimentos e atividades de ensino; a escolha dos procedimentos de avaliação. Embora essas questões não esgotem todos os fatores que envolvem o processo educativo, observou-se que quando as decisões mencionadas são tomadas de forma coerente e consciente pelo professor, tem-se uma boa estruturação deste processo de ensino- aprendizagem. Essa experiência de aprendizagem, num processo permeado por afetos alegres (no sentido espinosano do termo), não só possibilita que os estudantes se apropriem dos conteúdos, mas também contribui para uma aproximação entre estudantes e objeto de conhecimento (LEITE, 2018).

Quando se trata do ensino-aprendizagem de uma língua, há algumas especificidades que precisam ser discutidas. A concepção de ser humano aqui assumida, com base em Vigotski (1934, 1960), compreende que a linguagem é fundamental para o desenvolvimento das funções psíquicas e da subjetividade, sendo esta considerada o principal sistema simbólico da humanidade. Da mesma forma, a concepção discursiva de linguagem (BAKHTIN, 1929) adotada indica a linguagem como constitutiva da subjetividade humana. Esta relação entre linguagem - em 
seu caráter discursivo e dialógico - e subjetividade deve ser tomada como objeto de estudos das pesquisas sobre afetividade no ensino de línguas.

Bakhtin (1929, p. 205) afirma que a palavra é "a ponte que liga o eu ao outro" e é "a ponte basilar da ciência das ideologias" (BAKTHIN, 1929, p. 101). Vigotski (1934) assume que a palavra realiza a experiência e afirma que a palavra é o "microcosmo da consciência" (VIGOTSKI, 1934, p. 486). Com base em tais concepções de linguagem, não se poderia resumir a aprendizagem de línguas a meras atitudes decodificadoras pelos estudantes. Smolka (1988, p. 45) destaca que a língua(gem) não é apenas um objeto de conhecimento, mas também "constitutiva do conhecimento na interação". Nesse sentido, a dimensão afetiva no processo de ensino-aprendizagem de língua extrapola a questão da produção de conhecimento e abarca também a questão discursiva.

No estudo dos afetos no processo de ensino-aprendizagem de língua, percebe-se que o espaço para a produção dialógica de sentidos é fundamental para a apropriação dos conteúdos, conforme indicam Quast e Banks-Leite (2011), bem como para a aproximação dos estudantes com a língua (ORLANDO, 2019). Os sentidos vão sendo tecidos por meio das relações e prática sociais, em um processo permeado por emoções. Desta forma, argumenta-se que a educação linguística crítica - que propicie ao estudante a oportunidade de refletir sobre sua própria língua, sua identidade e sua cultura -, além de ter caráter formador, pode também contribuir para construção de boas relações, isto é, marcadas por afetos alegres, entre estudantes e a língua inglesa enquanto objeto de conhecimento.

\section{ASPECTOS METODOLÓGICOS/CONTEXTO}

Os dados apresentados neste artigo provêm de pesquisa de mestrado² (ORLANDO, 2019), do tipo qualitativa e de caráter etnográfico. A pesquisa foi realizada em um centro de línguas de uma universidade estadual paulista, em uma turma de Inglês IV. A pesquisadora acompanhou o desenvolvimento da disciplina durante todo o semestre, realizando observações, videogravações das aulas e anotações em diário de campo. Além disso, eram realizadas minientrevistas com os estudantes, antes ou após as aulas, para se obter dados acerca de suas impressões sobre as aulas. Ao final do semestre, foi realizada também uma entrevista com a professora da turma, na qual foram abordados aspectos de sua prática pedagógica e de sua formação como docente. A turma era composta por vinte e três estudantes, de diversos cursos da graduação e da pós-graduação, sendo que todos cursavam a disciplina de inglês em caráter não-obrigatório. A professora Lúcia ${ }^{3}$, responsável pelo curso, tinha 62 anos e trabalhava como professora colaboradora na instituição. Ela era formada em Letras Inglês-Português e tinha mestrado em Linguística Aplicada.

Os dados gerados na pesquisa de campo foram organizados em atividade de ensino, uma escolha que se deveu à percepção da pesquisadora, ainda em campo, de que a professora Lúcia desenvolvia uma grande variedade de atividades. Com base em Garzella (2013), entende-se que a atividade de ensino é um processo que se desenvolve na relação entre professor e aluno, englobando três aspectos distintos: a) instrução, que diz respeito às instruções fornecidas pelo professor ou por um colega, para a realização de uma atividade pelo estudante; b) desenvolvimento, que se refere à execução da atividade pelo estudante e onde se preveem intervenções necessárias e adequadas do professor; c) feedback, que consiste no retorno do professor sobre a atividade realizada pelo aluno, que pode incluir correções, informações, incentivos, todas com objetivo de levar o estudante a compreender a qualidade de seu trabalho. Esse modelo representa um parâmetro teórico para análise, mas ressalta-se que, na dinâmica da sala de aula, estes três momentos não ocorrem de maneira linear (GARZELLA, 2013).

Com este construto sobre atividade de ensino, realizou-se a análise dos dados que resultou na identificação e análise de dez tipos de atividades de ensino. Neste artigo, o foco será a atividade nomeada "Workshop 'Saving Indegenous Languages in Western Amazonia". A partir da descrição dessa experiência, serão analisas suas implicações afetivas na relação entre estudantes e língua inglesa. Além disso, serão tecidas considerações sobre as contribuições desta atividade para uma educação linguística crítica.

2. Financiada pela FAPESP (Processo n ${ }^{\circ} 17 / 00588-9$ ); aprovado pelo Comitê de Ética da Unicamp (Número do CAAE: 69384017.1.0000.5404).

3. Foram utilizados nomes fictícios para se preservar a identidade dos participantes. 


\section{WORKSHOP "SAVING INDEGENOUS LANGUAGES IN WESTERN AMAZONIA"}

No centro de línguas, era adotado um livro didático para os cursos de inglês. No entanto, a professora Lúcia utilizava materiais extras variados em suas aulas, como trechos de filmes, músicas, notícias, textos postados em redes sociais, entre outros, o que era muito apreciado pelos estudantes. No livro didático, na unidade com o tema "Language and learning", havia um texto sobre línguas que corriam risco de desaparecer. Nele eram abordados alguns dos motivos para essa situação, bem como eram descritas algumas iniciativas para se preservar essas línguas. A partir disto, a professora Lúcia decidiu expandir a discussão sobre a temática e convidou uma pesquisadora sobre línguas indígenas brasileiras para dar uma palestra na aula. Essa estudiosa contou sobre projeto de revitalização de línguas indígenas no Acre, fazendo uma introdução sobre aspectos históricos e políticos da situação dessas línguas no Brasil.

Para dar continuidade aos temas abordados na palestra, a professora Lúcia preparou uma tarefa em que os estudantes deveriam produzir um texto sobre essa experiência. Dessa forma, como disse a professora Lúcia, o que seria apenas uma palestra foi transformado em um workshop, pois os estudantes puderam refletir e elaborar sobre as questões que estavam em pauta. Em consonância com a fala da professora, entende-se que o fato de os estudantes terem realizado produções fez com que essa experiência se configurasse como uma atividade de ensino. Isto porque três elementos somaram-se à palestra: as instruções da professora para produção do texto; o desenvolvimento da atividade pelos estudantes, concretizado nas produções textuais; o retorno da professora, que se deu por meio da correção dos textos e de feedbacks transmitidos oralmente, em sala de aula.

Por meio dos textos, é possível obter um relato da palestra, o que contextualiza a atividade. Além disso, por se ter acesso às reflexões dos estudantes, pode-se analisar como esta atividade os afetou. A seguir, serão apresentados trechos dos textos ${ }^{4}$ dos estudantes, organizados de acordo seu conteúdo, de forma que serão expostos os seguintes tópicos: relatos do workshop; reflexões sobre Brasil, língua e cultura; reflexões sobre a própria aprendizagem.

\subsection{Relatos do workshop}

Nesta seção, estão agrupados trechos dos textos dos estudantes em que eles descrevem e relatam a palestra. Estas informações permitem uma contextualização da atividade. Os excertos a seguir trazem informações sobre a palestrante e sobre o tema do workshop.

The workshop "Saving' Indigenous Languages in Western Amazonia" was conducted by Professor T. in on October 26" 2017. The Professor talked about her experience working with the Comissão Pró-Índio do Acre (CPI-Acre), a non-profit organization that works to "save" indigenous languages in occidental Amazonia. (Trecho de texto escrito por Júlia)

T. talked about the mith myth of Brazil as a monolingual country. She compared the number of indigenous peoples and indigenous languages in the country at the beginning of the colonial era and nowadays. She talked about Nheengatu, the main Brazilian language before during colonization, and also about Marquis de Pompal's Language Policy. (Trecho de texto escrito por Giovana)

On October 26, 2017, during the English IV class at CL, Professor T. PhD conducted the workshop: "Saving' Indigenous Languages in Western Amazonia" where, within its line of applied research in Applied Linguistics. She presented, an overview of indigenous languages in Brazil and how they were modified, lost and how to keep them alive in the country today. (Trecho de texto escrito por Carolina).

A partir destes excertos, pode-se visualizar melhor como ocorreu a palestra e quais assuntos foram discutidos. A seguir, o relato de Carolina mostra com clareza como foi estruturada a palestra, indicando os conteúdos de cada uma de suas partes. Primeiramente, a professora convidada introduziu a história dos indígenas no Brasil e problematizou a questão sobre como eles vivem atualmente. Em seguida, tratou-se da história do Brasil, com foco em detalhes que afetaram os indígenas e suas línguas. Por fim, foi exibido parte de um documentário produzido por indígenas em que se conta a história deles.

The workshop began with an introduction about indigenous tribes in Brazil and how the indigenous people look like and act currently. (...)

In the second part of the Workshop, Professor T. told us about some details of Brazilian history that have affected indigenous languages directlyin indigenous languages. Lastly Finally, she explained about Comissão Pró Índio do Acre (CPI-AC) and some projects that she developed in occidental Amazonia during her period as member of CPI-AC.

4. Todos dados apresentados são provenientes da pesquisa de Orlando (2019). Os trechos dos textos estão reproduzidos aqui da forma como a professora Lúcia os entregou para os estudantes após a correção, sendo apresentados em inglês, pois considera-se que a qualidade da produção dos estudantes também é um dado relevante para o artigo. As únicas alterações feitas pela pesquisadora foi a retirada de informações que poderia levar à identificação dos participantes. 
In addition, the film named "I've already beeame become an image" was showed to us. The film was very interesting and also different, because it was all made by indigenous people. Besides, it shows the indigenous view about all their history. (Trecho de texto escrito por Maria)

As instruções fornecidas pela professora Lúcia para a escrita do texto solicitavam que os alunos escrevessem um resumo das informações apresentadas no workshop. O relato destes trechos se faz relevante pelo fato de terem sido informações novas para os estudantes e também porque, posteriormente, serão discutidas as reflexões que eles fizeram a partir do conteúdo da palestra. Além disso, é interessante notar que, em suas produções, cada estudante enfatizou diferentes aspectos do conteúdo apresentado em sala, como se pode notar a seguir:

Tabela 1. Trechos sobre conteúdos da palestra

\begin{tabular}{|c|c|}
\hline $\begin{array}{l}\text { Joyce comenta as informações sobre multilinguismo } \\
\text { no Brasil }\end{array}$ & $\begin{array}{l}\text { First, she introduced the workshop approaching multilinguism } \\
\text { multilingualism in Brazil: there are more than } 188 \text { indigenous languages, } \\
\text { but in } 1500 \text { it was there were around } 1300 \text {, which can be explained } \\
\text { by the fact that in } 1758 \text {, Portuguese has beeome became the national } \\
\text { language. Until the end of the } 1980 \text { s and up the } 80 \text { 's it was forbidden } \\
\text { to speak any indigenous languages }\end{array}$ \\
\hline $\begin{array}{l}\text { Miguel traz a informação de que muitos indígenas } \\
\text { vivem em áreas urbanas e comenta sobre as } \\
\text { dificuldades enfrentadas pelos povos indígenas no } \\
\text { Brasil }\end{array}$ & $\begin{array}{l}\text { Đuring Throughout the Brazilian history, the indigenous people } \\
\text { faced various problems with the society. Problems like not to being } \\
\text { recognized as an indigenous or preeoneeptionabout prejudice against } \\
\text { their ethnicity are common. About } 36,2 \% \text { of the indigenous society } \\
\text { are living in urban areas; their relationship with the "white man" even } \\
\text { being constant is constantly fraught with prejudice, which makes their } \\
\text { life difficult. }\end{array}$ \\
\hline $\begin{array}{l}\text { A estudante Laís relata o trabalho realizado pela } \\
\text { professora T. na revitalização das línguas indígenas } \\
\text { no Acre. }\end{array}$ & $\begin{array}{l}\text { The teacher continued the workshop telling us about her personal } \\
\text { experience in Pró-Indian Acre Commission. She has acted as a teacher } \\
\text { in a language revitalization program. She told us stories of a lot of strong } \\
\text { people who wants to recover the linguistic knowledges and transmit it } \\
\text { to new generations. She has realized that it can get bring back the self- } \\
\text { esteem for communities, helping them to hold on their political rights. }\end{array}$ \\
\hline
\end{tabular}

Considerando que esse workshop foi uma atividade no contexto do curso de inglês observado para a pesquisa, é válido analisar e discutir como esta experiência se adequa ao construto de atividade ensino (GARZELLA, 2013) apresentado anteriormente. Portanto, serão destacados os três momentos que compõem a atividade, a saber: instrução, desenvolvimento e feedback. Foi possível observar que a professora apresentou instruções claras sobre a tarefa que os estudantes deveriam realizar. Foi enviado aos estudantes um documento com instruções escritas, que indicava alguns tópicos que deveriam ser abordados. Essas instruções foram claras e bem detalhadas, sendo que a professora forneceu dicas para facilitar o trabalho dos estudantes. Além de entregar este documento aos estudantes e postá-lo na plataforma virtual, ela também explicou, em sala de aula, o que era esperado que eles fizessem.

Conforme lembra Garzella (2013), uma atividade de ensino pode ter problemas se as instruções não forem claras, pois isto pode dificultar o desenvolvimento da proposta pelos estudantes. Dessa forma, destaca-se que o cuidado e atenção da professora Lúcia para este ponto foi significativa para o bom desempenho dos alunos. Da mesma forma, o feedback após o desenvolvimento da atividade pelos estudantes também é imprescindível. Somente por meio do feedback que o estudante tem a possibilidade de rever seus erros e superá-los. No âmbito do workshop, a professora forneceu feedback detalhado, em que ela apontou correções para melhoria do texto, além de fazer comentários e elogios.

\subsection{Reflexões sobre Brasil, língua e cultura}

Conforme foi mencionado anteriormente, com a palestra, os estudantes aprenderam muitas informações novas sobre a história do Brasil e dos povos indígenas. Nos textos que escreveram, foi possível observar reflexões acerca deste novo conhecimento. A partir das informações trazidas sobre a quantidade de línguas existentes no país, bem 
como sobre a história de políticas linguísticas que buscaram impor o português como língua única, os estudantes puderam aprender que o Brasil é um país multilíngue e compreender a relação entre língua e cultura:

Tabela 2. Trechos sobre relações entre língua e cultura

\begin{tabular}{|l|l|}
\hline $\begin{array}{l}\text { Laís comenta sobre a pluralidade de línguas e culturas } \\
\text { que compõem o Brasil. }\end{array}$ & $\begin{array}{l}(\ldots) \text { the workshop could show me a very different perspective from of } \\
\text { Brazil. T. has showed me a pluricultural and plurinational Brazil. Besides } \\
\text { that, we could realized how the language is an important instrument for } \\
\text { a people's identity, self-esteem and fight for their own rights. It was } \\
\text { remarkable! }\end{array}$ \\
\hline $\begin{array}{l}\text { Giovana ressalta a importância de se "salvar" uma } \\
\text { língua. }\end{array}$ & $\begin{array}{l}\text { The workshop was very interesting. It made me realize how important } \\
\text { it is to 'save' a language and made me notice it's just a battle of a much } \\
\text { larger war. Keeping a language is part of the process of keeping a culture. }\end{array}$ \\
\hline $\begin{array}{l}\text { Isadora indica que a manutenção da língua está ligada } \\
\text { à identidade de um povo. A estudante associa a luta } \\
\text { pela língua a outras lutas, por direitos e respeito. }\end{array}$ & $\begin{array}{l}\text { Finally, saving the indigenous language means giving an ethnic identity, } \\
\text { so that they identify themselves as Indians. The language shows that } \\
\text { they exist. To lose a language is to lose part of the cultural construction. } \\
\text { Saving a language is just a little bit of the indigenous world, so they can } \\
\text { get the rights and respect. That's the why the workshop was so good, } \\
\text { the Professor show us the importance of the traditional people like as } \\
\text { was previously said. }\end{array}$ \\
\hline
\end{tabular}

Os trechos acima indicam que os estudantes aprenderam sobre a relação entre língua, identidade e cultura, bem como sobre a importância de se preservar as línguas indígenas. Com isto, a sala de aula de língua inglesa deslocou-se do discurso hegemônico que coloca a língua inglesa como língua global para dar espaço às línguas minoritárias.

Além das reflexões sobre as relações entre língua, cultura e identidade, os estudantes mostraram também que conhecer este tema foi importante para a própria formação geral, porque passaram a entender melhor a história e a cultura de seu próprio país.

\section{Tabela 3. Trechos com reflexões sobre história e cultura do Brasil}

\begin{tabular}{|c|c|}
\hline $\begin{array}{l}\text { Júlia demonstra ter apreciado conhecer mais sobre } \\
\text { história e cultura de seu país. }\end{array}$ & $\begin{array}{l}\text { I liked the workshop very much because I had the opportunity to learn } \\
\text { more about the culture of my own country. }\end{array}$ \\
\hline $\begin{array}{l}\text { Mariana reflete sobre como é comum se ignorar a } \\
\text { história de luta e violências dos povos indígenas. } \\
\text { A estudante ressalta a importância de se lutar pela } \\
\text { identidade diante de tanta violência. }\end{array}$ & $\begin{array}{l}\text { I learned more about the importance of the language and the Indian's } \\
\text { culture. The presentation made me think how much we are ignorant } \\
\text { about understanding other people and how important it is for these } \\
\text { people to have their own identity after a long time of violence. }\end{array}$ \\
\hline $\begin{array}{l}\text { Miguel comenta que este tema é pouco tratado e } \\
\text { defende a importância de que seja mais amplamente } \\
\text { discutido. }\end{array}$ & $\begin{array}{l}\text { The workshop was very interesting since this theme is not much } \\
\text { discussed, which makes us alienated. Thus, it is extremely important } \\
\text { that this theme be discussed more closely with every society. }\end{array}$ \\
\hline $\begin{array}{l}\text { Milena destaca que a formação do Brasil atual se } \\
\text { deu por meio de políticas opressoras. A estudante } \\
\text { defende que se promovam os direitos dos povos } \\
\text { indígenas. }\end{array}$ & $\begin{array}{l}\text { I think the workshop was very interesting and important to know about } \\
\text { our history as a country and how oppressive policies have built Brazil } \\
\text { as we know it nowadays. It is important to promote the respect of the } \\
\text { Indigenous people's rights and to recognize the their importance-of } \\
\text { them in the formation of the Brazilian people }\end{array}$ \\
\hline
\end{tabular}

Os excertos apresentados acima sugerem que os estudantes aprenderam (e apreciaram aprender) sobre a história dos povos e línguas indígenas no Brasil, um percurso marcado por violência e processos de exclusão. Siqueira (2018) destaca a importância de que o ensino de línguas, para que se configure como uma educação linguística crítica, desenvolva a capacidade de os estudantes pensarem criticamente sobre a realidade que os cerca. Aprender sobre a história de seu país, especialmente uma história que é apagada dos currículos, pode contribuir para o desenvolvimento desse pensamento crítico. 
Pode-se notar que muitos estudantes demonstraram engajamento político, indicando a necessidade e importância das lutas dos povos indígenas. Com relação a isto, destaca-se o texto de Maria, em que a aluna menciona se sentir mais preparada para discutir políticas afirmativas:

I really enjoyed to had participated participating in these this workshop, because it gave to me much more knowledge about this theme. From now on I feel that I have more basis appropriate knowledge to discuss policies of inclusion of indigenous people, including policies in universities. (Trecho de texto escrito por Maria).

No momento em que a pesquisa estava sendo realizada, a universidade estudava mudanças em seu vestibular e, a partir do ano seguinte, adotou, entre outras medidas, o vestibular indígena. Portanto, observa-se a relevância do tema, discutido em sala de aula, com a realidade vivida por estes universitários.

Neste sentido, pode-se afirmar que esta atividade contribuiu para uma formação cidadã (ROCHA, 2012), pois os estudantes aprenderam sobre temáticas que influenciam na participação social de ambientes que eles ocupam. E, como lembram Osler \& Starkey (2000), as questões raciais e o racismo são barreiras no exercício da cidadania, portanto, ressalta-se a relevância dos temas tratados no workshop.

\subsection{Reflexões sobre a própria aprendizagem}

As pesquisas sobre afetividade têm indicado que um fator importante para a aproximação do estudante com o objeto de conhecimento é a sensação de "sentir-se capaz de", isto é, a percepção do aprendiz sobre os avanços e ganhos em sua própria aprendizagem (LEITE, 2018). A experiência do workshop foi bastante favorável neste sentido, pois os estudantes tiveram a oportunidade de ouvir uma palestra conduzida na língua inglesa e, então, perceber suas dificuldades e seus pontos fortes. Os trechos desta seção ilustram este fenômeno.

Tabela 4. Reflexões sobre compreensão da palestra

\begin{tabular}{|l|l|}
\hline $\begin{array}{l}\text { Joyce destacou que a experiência foi incrível por } \\
\text { proporcionar o uso da língua inglesa em um contexto } \\
\text { "natural" (não artificial). }\end{array}$ & $\begin{array}{l}\text { Attending a workshop in English is an amazing experience, because the } \\
\text { use of English in this situation becomes natural and interesting, and it is } \\
\text { not so difficult because the context helps us to understand and explore } \\
\text { the language. }\end{array}$ \\
\hline $\begin{array}{l}\text { Giovana relatou que não foi fácil acompanhar a } \\
\text { discussão conduzida em inglês, mas ter conseguido } \\
\text { entender a conversa foi recompensador. }\end{array}$ & $\begin{array}{l}\text { The experience of attending an English workshop was challenging. } \\
\text { However, I was able to understand the whole conversation, video and } \\
\text { slides. It was rewarding! }\end{array}$ \\
\hline $\begin{array}{l}\text { Diego demonstrou-se satisfeito porter compreendido } \\
\text { a discussão sobre um tema que era novo para ele. }\end{array}$ & $\begin{array}{l}\text { Iliked the workshop, because I saw another person speaking in English } \\
\text { for two hours, and it wasn't a theme that I knew anything before the } \\
\text { workshop. I learned many things about the theme, and I'm happy with } \\
\text { about it. }\end{array}$ \\
\hline $\begin{array}{l}\text { Isadora relatou ter tido alguma dificuldade para } \\
\text { acompanhar a discussão, mas avalia positivamente a } \\
\text { experiência. A estudante considerou que participar } \\
\text { de uma palestra em inglês pode promover a } \\
\text { aprendizagem da língua. }\end{array}$ & $\begin{array}{l}\text { The experience of having participated in the workshop was very good, } \\
\text { lhe understanding was so good too, although in a few hours get a little } \\
\text { lost especially in the part of the documentary. Overall, the experience } \\
\text { helps with the understanding and reasoning in English. }\end{array}$ \\
\hline
\end{tabular}

Ressalta-se que os estudantes relataram terem encontrado alguns obstáculos para acompanhar uma palestra conduzida na língua inglesa. No entanto, o fato de terem sucesso nesta tarefa fez com que eles se sentissem orgulhosos e felizes. Alguns estudantes deram maior ênfase na dificuldade de compreender o workshop, mas é válido notar que, mesmo nestes casos, houve reflexão sobre o próprio aprendizado da língua, como foi o caso de Carolina.

As a personal analysis of the workshop, I found the topic chosen very interesting, but I had difficulties in aecompanying to understand the vocabulary, expressions and some sentences that I understood by because of the context in which it was inserted. (Trecho de texto escrito por Carolina). 
Esta estudante relatou não ter entendido algumas expressões e palavras, contudo, ela indicou que conseguiu compreendê-las pelo contexto da discussão. Enfatiza-se, neste caso, que a estudante demonstrou, de forma consciente, uma estratégia para aprendizagem e uso da língua inglesa.

As relações entre estudante e objeto de conhecimento são socialmente construídas em um processo histórico das práticas vivenciadas pelo aprendiz. A percepção de que determinada experiência proporcionou a apropriação de novos conhecimentos tem implicações positivas na relação sujeito-objeto de conhecimento (LEITE, 2018). No caso do workshop, os estudantes perceberam o sucesso de seu aprendizado na língua inglesa e, além disso, puderam sentir que se apropriavam de novos conhecimentos sobre as línguas indígenas brasileiras. Os sentimentosemoções presentificadas (SAWAIA e MAGIOLINO, 2016) - dos estudantes em relação à atividade são constituídos predominantemente de afetos alegres, o que aumenta a potência de agir (SPINOZA, 2017) destes estudantes no contexto do processo de ensino-aprendizagem. Portanto, ressalta-se que o workshop organizado pela professora Lúcia possibilitou não somente o desenvolvimento da criticidade dos estudantes, mas também promoveu a aproximação entre estudantes e língua inglesa.

\section{CONSIDERAÇÕES FINAIS}

Este trabalho não pretende, de forma alguma, encerrar a questão das relações étnico-raciais no ensino de línguas. Deve-se considerar que a experiência aqui descrita foi facilitada pelo fato de ter acontecido em um centro de línguas universitário, o que possibilitou o acesso a uma pesquisadora da área de línguas indígenas. No entanto, considera-se que a experiência foi aqui relatada foi positiva e pode ser inspiradora para professores de línguas.

Além disto, considerando que uma ideia não muda um afeto (SPINOZA, 1677), defende-se a necessidade da discussão sobre afetividade no contexto da educação linguística crítica. Práticas de ensino que se centram na dimensão cognitiva e ignoram as emoções e sentimentos dos educandos dificilmente promoverão mudanças de atitudes acerca das desigualdades sociais. Portanto, a discussão apresentada pode contribuir para a construção de novas práticas educativas emancipadoras, que levem em consideração a dimensão afetiva do desenvolvimento humano e do processo de ensino-aprendizagem

Neste sentido, destaca-se o caráter sempre social das emoções (VIGOTSKI, 1934, 1960; SWAIN, 2013) construídas nas e pelas relações sociais. Portanto, é no diálogo e nas interações que as emoções vão sendo significadas pelos sujeitos. Com isso, é possível compreender que as discussões e o confrontamento entre opiniões diversas são carregados de conteúdos afetivos (e não somente cognitivos), o que pode levar à revisão de posicionamentos pessoais diante do novo que é apresentado pelo interlocutor

Por fim, evidencia-se que o workshop sobre línguas indígenas proporcionou o desenvolvimento da criticidade dos estudantes acerca de questões étnicas. Além disso, reconhecendo-se a importância de que os estudantes de língua sejam autores de suas próprias palavras (QUAST E BANKS-LEITE, 2011), ressalta-se que a produção escrita dos estudantes possibilitou que eles tomassem a posição de autoria, produzindo sentidos e (re)elaborando aquilo que havia sido discutido na palestra.

\section{REFERÊNCIAS}

ALMEIDA, L. R. e MAHONEY, A. A. (2014). Afetividade e aprendizagem: contribuições de Henri Wallon. São Paulo: Edições Loyola.

BAHKHTIN, M.M (VOLÓCHINOV, V.) (1929). Marxismo e filossfia da linguagem: problemas fundamentais do método sociológico da ciência da linguagem. Tradução de Sheila Grillo. São Paulo: Editora 34, 2017.

CAMARGO, M.i FERREIRA, A. de J. (2012). O PROFESSOR DE LÍNGUA INGLESA E O ALUNO QUILOMBOLA: letramento crítico e formação de professores. Revista da Associação Brasileira de Pesquisadores/as Negros/as (ABPN), [S.1.], v. 4, n. 8, p. 192-210, out. 2012. ISSN 2177-2770. Disponível em: < http://abpnrevista.org.br/revista/index.php/revistaabpn1/ article/view/260>. Acesso em: 13 maio 2020.

FEITOSA, C. F. J. (2012). "Aqui tem racismo!": um estudo das representações sociais e das identidades das crianças negras. Dissertação de mestrado em Educação. Universidade Estadual de Campinas, Campinas, SP, Brasil. 
FERREIRA, A. de J. (2007). What has race/ethnicity got to do with EFL teaching? Linguagem e Educação 10: 211-233.

GARZELLA, F. A. C. (2013). A disciplina de cálculo 1: análise das relações entre as práticas pedagógicas do professor e seus impactos no aluno. Tese de Doutorado, Faculdade de Educação, UNICAMP. Campinas, SP.

JORGE, M. L.S. Preconceito contra o ensino de língua estrangeira na rede pública. In: LIMA, D.C. (org) Ensino e aprendizagem de lingua inglesa-conversas com especialistas. São Paulo: Parábola Educacional, 2009

LEITE, S.A. (2012). Afetividade nas práticas pedagógicas. Temas em Psicologia. Sociedade Brasileira de Psicologia, vol 20, no. 2, 355-368

LEITE, S. A. (2018). Afetividade: as marcas do professor inesquecível (org.). Campinas, SP: Mercado de Letras.

MAGIOLINO, L. L. S.; SMOLKA, A. L. B. (2013) How Do Emotions Signify? Social Relations and Psychological Functions in the Dramatic Constitution of Subjects, Mind, Culture, and Activity, 20:1, 96-112, DOI: 10.1080/10749039.2012.743155

MAHONEY \& ALMEIDA (2004) (orgs) A constituição da pessoa na proposta de Henri Wallon. São Paulo: Edições Loyola.

MOURA, G. (2005). O direito à diferença. In: MUNANGA, K. (org). (2005). Superando o racismo na escola. Brasília: Ministério da Educação, Secretaria de Educação Continuada, Alfabetização e Diversidade.

MUNANGA, K. (org). (2005). Superando o racismo na escola. Brasília: Ministério da Educação, Secretaria de Educação Continuada, Alfabetização e Diversidade.

NOGUEIRA, A.L.H. (2010). As normas e as práticas discursivas na relações de ensino. In: Smolka, A. L. B.; Nogueira, A. L. H. (org.), Questões de desenvolvimento bumano: práticas e sentidos. Campinas: Mercado de Letras, p.57-80.

ORLANDO, I R. (2019). Afetividade e ensino de língua inglesa: um olhar para as práticas pedagógicas. Dissertação (mestrado) - Universidade Estadual de Campinas, Faculdade de Educação, Campinas, SP. Disponível em: http://www.repositorio.unicamp.br/ handle/REPOSIP/334405. Acesso em: 29 Mar. 2021.

OSLER, A.; STARKEY, H. (2000). Intercultural education and FL learning: issues of racism, identity, and modernity. Race, Ethnicity and Education 3: 207-221.

PENNYCOOK, A. (1994). The cultural politics of English as an international language. Nova York: Longman.

QUAST, K. e BANKS-LEITE, L. (2011). Da linguagem ao discurso: memória de práticas e ensino de língua estrangeira. In: SMOLKA, A.L.B.S. e NOGUEIRA, A.L.H. (org) Emoção, memória, imaginação: a constituição do desenvolvimento bumano na bistória e na cultura._Campinas: Mercado de Letras.

RAJAGOPALAN. N, K. (2003). Por uma linguística crítica: linguagem, identidade e a questão ética. São Paulo: Parábola Editorial.

RAJAGOPALAN, K. (2004). The Concept of "World English" and its Implication for ELT. In: ELT Journal. v. 58, n. 2, Oxford: Oxford University Press, pp. 111-117

ROCHA, H, C. (2012). Língua inglesa e contexto acadêmico-universitário: avaliando e reprojetando práticas plurilíngues e transculturais sob a luz dos multiletramentos. Revista Contemporânea de Educação, Rio de Janeiro, vol.7, n. 13, janeiro/ julho 2012.

ROCHA, C. H.; MACIEL, R. F. (Orgs.). (2013) Língua Estrangeira e Formação Cidadãa: Por entre Discursos e Práticas. Campinas, SP: Pontes Editores.

RODRIGUES, A. L. (2009) A língua inglesa na África: opressão, negociação e resistência. Campinas: Editora da Unicamp.

SAWAIA, B. B.(2000). Emoção como locus da produção do conhecimento - Uma reflexão inspirada em Vygotsky e no seu diálogo com Espinosa. In III Conference for Sociocultural Research, 1. Campinas: Universidade Estadual de Campinas. 
SAWAIA, B. B. (2018). Prólogo. In: SAWAIA, B. B.; ALBUQUERQUE, R.; BUSARELLO, F. R. (2018). Afeto \& Comum: reflexões sobre a práxis psicossocial. São Paulo: Alexa Cultural.

SAWAIA, B. B.; MAGIOLINO, L.L.S. (2016). As nuances da afetividade: emoção, sentimento e paixão em perspectiva. In: Diálogos na perspectiva bistórico-cultural: interlocuções com a clínica da atividade / Luci Banks-Leite, Ana Luiza B. Smolka, Daniela Dias dos Anjos, (organizadoras). Campinas, SP: Mercado de Letras

SIQUEIRA, S. (2018). Por uma educação linguística crítica. In: PESSOA, R. R.; SILVESTRE, V. P. V.; MONTE MÓR, W. (2018). Perspectivas críticas de educação linguística no Brasil: trajetórias de professoras/es universitárias/os de inglês. São Paulo: Pá de palavra.

SMOLKA, A. L. B. (1988). A criança na fase inicial da escrita: a alfabetização como processo discursivo. São Paulo: Cortez, 2008.

SPINOZA, B. de. (1677). Ética. São Paulo: Autêntica, 2013.

SWAIN, M. (2013). The inseparability of cognition and emotion in second language learning. Language Teaching, 46, pp 195-207. Cambridge: Cambridge University Press.

SWAIN, M.; KINNEAR, P.; STEINMAN, L. (2011). Sociocultural Theory in Second Language Education: An introduction through narratives. Edição ebook Kindle. Bristol: Multilingual Matters.

VIGOTSKI, L. S. (1934). A Construção do Pensamento e da Linguagem. Tradução Paulo Bezerra. São Paulo: Martins Fontes, 2009

VIGOTSKI, L. S. (1960). Obras escogidas. Tomo III. Madrid: Machado Grupo de Distribución, 2012. 5 v.

WALLON, H. (1941). A evolução psicológica da criança. São Paulo: Martins Fontes, 2007.

WALLON, H. (1942). Do ato ao pensamento. Rio de Janeiro: Editora Vozes, 2008.

Recebido: 30/10/2020

Aceito: 8/3/2021

Publicado: 22/3/2021 